COPYRIGHT @ 2019 INTERNATIONAL JOURNAL OF SCIENCE DENTISTRY | AVAILABLE ONLINE http://www.periodicos.uff.br/index

\title{
ATENDIMENTO ODONTOLÓGICO AO PACIENTE PORTADOR DE HEMOFILIA C: QUAIS SÃO CUIDADOS NECESSÁRIOS PARA UM CORRETO ATENDIMENTO? - REVISÃO DE LITERATURA
}

\section{DENTAL CARE TO THE HEMOPHILIA C PATIENT: WHAT ARE THE NEEDS FOR THE CORRECT SERVICE? LITERATURE REVIEW.}

\section{Angela Figueiredo de Brito Resende}

Especialista em Pacientes com Necessidades Especiais pela Orthodontic / RJ.

Cirurgião - dentista aposentada do Serviço de Cirurgia Buco-Maxilo-Facial do Hospital Municipal Salgado Filho / RJ.

\section{Isabelly de Vasconcellos Pereira}

Graduada em Odontologia pela Universidade Federal Fluminense / RJ.

Pós-Graduanda do curso de especialização em Implantodontia da Universidade Federal Fluminense / RJ.

\section{Marcelo Ventura de Andrade}

Mestre em Odontopediatria pela São Leopoudo Mandic / SP.

Professor de Odontopediatria do Curso de Odontologia da Universidade Iguaçu / RJ.

\section{Suelen Cristina Sartoretto}

Doutora em Odontologia pela Universidade Federal Fluminense / RJ.

Professor de Cirurgia Bucal do Curso de Odontologia da Universidade Iguaçu / RJ.

\section{Rodrigo Figueiredo de Brito Resende}

Doutor em Odontologia pela Universidade Federal Fluminense / RJ.

Professor de Cirurgia Oral Menor da Faculdade de Odontologia da Universidade Federal Fluminense / RJ.

Universidade Federal Fluminense

Faculdade de Odontologia

Artigo de Revisão de literatura

\section{Rodrigo Figueiredo de Brito Resende}

Endereço: Rua Mario Santos Braga, 28 - Centro, Niterói - RJ, 24020-140

Telefone: (21) 997399282

E-mail: resende.r@hotmail.com 


\section{RESUMO}

A hemofilia é uma doença hemorrágica hemofilia hereditária ligada ao cromossomo X, caracterizada pela deficiência ou anormalidade da atividade coagulante do fator VIII (hemofilia A), do fator IX (B) ou mesmo do fator XI (hemofilia C). Esta doença é causada pela deficiência quantitativas dos fatores de coagulação, podendo causar sangramento espontâneo ou mesmo induzido por cirurgia ou trauma. A hemofilia C foi descrita em 1953, após vários membros de famílias de descendentes judaica (judeus Ashkenazi, descendentes de judeus que abandonaram Jerusalém e migraram para a Polônia e Báltico durante o primeiro século), terem surgidos com hemorragias excessiva ao serem submetidos a amigdalatomia e exodontias. A deficiência deste fator, é hereditária, resultante de uma mutação em um gene autossômico recessivo presente no cromossomo 4. Embora o diagnostico precoce seja de difícil execução, pois o paciente não apresenta sangramento espontâneo como em outros hemofilia, uma rigorosa anamnese deve ser realizada para que se possa tomar todas as medidas preventivas, evitando possíveis riscos ao paciente. O objetivo deste trabalho é realizar uma revisão de literatura sobre os cuidados necessários que o cirurgião - dentista deve ter frente a um paciente portador de hemofilia C. Para que o mesmo possa indicar e realizar corretamente o tratamento odontológico e evite possíveis riscos.

Palavras-chaves: Hemofilia; Hemofilia C; Atendimento odontológico; Fator XI. 
COPYRIGHT @ $\odot 2019$ INTERNATIONAL JOURNAL OF SCIENCE DENTISTRY | AVAILABLE ONLINE http://www.periodicos.uff.br/index

\begin{abstract}
Hemophilia is a hereditary hemorrhagic disease linked to the $\mathrm{X}$ chromosome, characterized by the deficiency or abnormality of factor VIII (hemophilia A), factor IX (hemophilia B) or even factor XI (hemophilia C) factor VIII coagulant activity. This disease is caused by the quantitative deficiency of coagulation factors, which can cause spontaneous bleeding or even induced by surgery or trauma. Haemophilia C was described in 1953 after several members of families of Jewish descent (Ashkenazi Jews, descendants of Jews who left Jerusalem and migrated to Poland and the Baltic during the first century), have arisen with excessive hemorrhages when undergoing tonsilatomy and Exodontia. The deficiency of this factor is hereditary, resulting from a mutation in an autosomal recessive gene present in chromosome 4. Although the early diagnosis is difficult to perform, since the patient does not present spontaneous bleeding as in other haemophilia, a rigorous anamnesis should be performed for That all preventive measures can be taken, avoiding possible risks to the patient. The objective of this work is to carry out a review of the literature on the necessary care that the dental surgeon must have in front of a patient with hemophilia $\mathrm{C}$. In order that he may indicate and correctly perform the dental treatment and avoid possible risks.
\end{abstract}

Key words: Hemophilia; Hemophilia C; Dental care; Fact 
COPYRIGHT @ 2019 INTERNATIONAL JOURNAL OF SCIENCE DENTISTRY | AVAILABLE ONLINE http://www.periodicos.uff.br/index

\section{INTRODUÇÃO}

A hemofilia é uma doença hemorrágica hereditária ligada ao cromossomo X, caracterizada pela deficiência ou anormalidade da atividade coagulante do fator VIII (hemofilia A), do fator IX (hemofilia B) ou mesmo do fator XI (hemofilia C) (MODOLO et al., 2010). Esta doença é causada pela deficiência quantitativas dos fatores de coagulação, podendo causar sangramento espontâneo ou mesmo induzido por cirurgia ou trauma (MODOLO et al., 2010; CONDE et al., 2007).

A deficiência do fator XI é uma doença hereditária, resultante de uma mutação em um gene autossômico recessivo presente no cromossomo (DIEGO, 2007). Essa mutação é rara na população em geral, afetando cerca de 1 pessoa em 1 milhão, mas sendo frequente em judeus Ashkenazi, afetanto 1 em cada 450 judeus Ashkenazi, cerca de $5 \%$ a $11 \%$. Já foram descritas mais de 180 mutações no gene do fator XI causadores da deficiência (CONDE et al., 2007; CAVALCANTE, 2004). A medida que o organismo produz uma menor quantidade do fator XI ou quando ele não funciona corretamente, a coagulação é inibida, não havendo formação de coagulo de fibrina (DIEGO, 2007). Este tipo difere das demais hemofilia mais conhecidas (A e B), por não causar hemorragia articular ou muscular e por acometer igualmente homens e mulheres (CAVALCANTE, 2004; DIEGO, 2007).

Normalmente, níveis de fator XI em torno de $30 \%$ são suficientes para o paciente ter uma vida normal, porém alguns doentes podem necessitar de níveis mais elevados. Alguns indivíduos que possuem um nível de fator XI plasmático extremamente baixo ou que foram expostos ao tratamento com plasma fresco congelado, podem desenvolver inibidores para esse fator. Desse modo, apresentam hemorragia grave durante e apos cirurgia, apesar da terapêutica com plasma (MODOLO et al., 2010; DIEGO, 2007).

No ano de 2011, foram registrados 112 casos no Brasil de hemofilia C. No mundo, no mesmo ano, 4759 casos. Essa analise foi realizada em 62 países, sendo $42 \%$ homens, $52 \%$ mulheres e $2 \%$, gênero não conhecido (DIAMENT, 2004).

Alguns estudos mostram que fator XI exerce uma importante ação na diminuição de fibrolise. Desse modo, pacientes com deficiência nesse fator tem maior predisposição a ter hemorragia em partes do corpo que possuem alta atividade fibrinolítica. Os tecidos mais propensos ao sangramento são: trato geniturinário, cavidade oral, principalmente apos extração dentaria, remoção das amigdalas e mucosa nasal (CAVALCANTE, 2004; DIAMENT, 2004). 
Afim de não colocarmos em risco a vida do paciente, uma rigorosa anamnese tem que ser realizada sempre. A deficiência do fator XI, é uma doença rara e, com isso, necessita de um diagnostico precoce, embora o mesmo seja de difícil execução, já que o paciente normalmente não sangram espontaneamente (EMILY et al., 2010).

Desse modo é necessário que seja feita a procura dessa deficiência nas populações mais afetadas como os judeus Ashkinazi e seus descendentes. Sendo necessário um tratamento eficiente e realizado de acordo com as características da deficiência de cada paciente, sendo ainda necessário um tratamento diferenciado quando ha presença de inibidores (CONDE et al., 2007; DIAMENT, 2004; EMILY et al., 2010).

\section{REVISÃO DA LITERATURA}

\section{Tipos de hemofilia}

A hemofilia é um distúrbio genético e hereditário que afeta a coagulação do sangue que é composto por várias substâncias, onde cada uma delas tem uma função.Algumas dessas substâncias são as proteínas denominadas de fatores da coagulação, que ajudam a hemostasia, quando ocorre o rompimento de vasos sanguíneos (CONDE et al., 2007).

Existem 13 tipos diferentes de fatores de coagulação e, seus nomes são expressos por algarismos romanos (Fator I ate o Fator XIII). Esses fatores são ativados quando ocorrem rompimento do vaso sanguíneo, num movimento de cascata. Quando ocorre uma deficiência quantitativa ou qualitativa dos fatores VIII, IX ou XI, a coagulação e interrompida antes da formação do coagulo, podendo levar a um sangramento mais demorado (CONDE et al., 2007; FERREIRA et al., 2010).

Segundo a Organização Mundial de Saúde (OMS), podemos classificar as hemofilias em quatro tipos distintos:

- Hemofilia A: Sendo a mais comum segundo a OMS, representando cerca de $80 \%$ dos casos, ocorre pela deficiência do Fator VIII;

- Hemofilia B: Ocorre pela deficiência do Fator IX;

- Hemofilia C: A mais rara e predominante numa etnia, ocorre pela deficiência do Fator XI;

- Hemofilia (A) Adquirida: Também chamada de Hemofilia A adquirida, que e uma alteração rara da coagulação sanguínea caracterizada pelo aparecimento

\section{A hemofilia C}

Também conhecida como Síndrome de Rosenthal, e envolvida na via intrínseca da cascata da coagulação. Costuma ser leve, mas pode assemelhar- se a hemofilia clássica. Sendo determinada por gene autossômico recessivo, não ligado ao cromossoma $\mathrm{X}$, portanto manifestase em homens e mulheres com mais frequência entre famílias judaicas descendentes de linhagens de judeus Asquenazi. Este tipo de hemofilia se difere dos tipos A e B, pois não leva a sangramento nas articulações. Além disso, costuma cursar com sangramento tardios no pós - operatório de procedimentos cirúrgicos (FERREIRA et al., 2010; MINISTÉRIO DA SAÚDE, 2015). 
COPYRIGHT @ 2019 INTERNATIONAL JOURNAL OF SCIENCE DENTISTRY | AVAILABLE ONLINE http://www.periodicos.uff.br/index

Pacientes portadores de hemofilia $\mathrm{C}$, requerem atenção e cuidados especiais ao serem atendidos pelos cirurgiões - dentistas, cuidados esses, que começam com uma minuciosa anamnese, associada a exame hematológico com solicitação de hemograma e coagulograma completos, além de exame intra - bucal e radiográfico quando necessário (MINISTÉRIO DA SAÚDE, 2015).

Quando detectado o transtorno de coagulação sanguínea, o cirurgião dentista deve estar preparado para oferecer o tratamento adequado a esses pacientes que, dependera da severidade da doença e do tipo de procedimento a ser realizado. Os procedimentos menos invasivos, podem ser realizados rotineiramente, obedecendo cuidados básicos. Porém quando incluem procedimentos cirúrgicos maiores, deve se tomar algumas precauções afim de diminuir os riscos hemorrágicos e suas complicações, além da consulta prévia com o hematologista responsável pelo paciente (FERREIRA et al., 2010; PEYVANDI et al., 2011).

\section{Como chegar ao diagnóstico?}

Nas décadas de 80 a 90, o diagnostico só era feito quando havia alguma hemorragia após lesão ou quando TTPA (tempo de tromboplastina parcial ativada), era prolongado e o teste da mistura após diagnosticar um TTPA prolongado, esse vai avaliar qual o motivo do prolongamento, se e a deficiência de um fator ou a presença de inibidores. Esses diagnósticos levavam apenas a uma hipótese de deficiência de fator XI. Nos dias atuais, o diagnostico e feito a partir da dosagem de fator XI no plasma por métodos imunológicos, como o ELISA, tendo como resultado níveis menores que 80 UI / Dl (CAVALCANTE, 2004; DIAMENT, 2004; FERREIRA et al., 2010).

A deficiência de fator XI tem como característica laboratorial um TTPA prolongado e um tempo de protrombina (TP) e de trombina (TT) normais. De todo modo, só é possível constatar a presença dessa doença se for descartada a doença de von Willebrand e hemofilia A e B. É possível diferenciar da deficiência de fator XI, já que esses estarão em baixa quantidade se a hemofilia A ou B estiver presente (PEYVANDI et al., 2011; COPPOLA et al., 2013).

Já a doença de von Willebrand e diferenciada da deficiência de fator XI também pelo nível de fator VIII e pela dosagem do antígeno do fator de von Willebrand (COPPOLA et al., 2013). Pacientes com nível extremamente baixo de fator XI no plasma (cerca de 1 UI/ DL) expostos a terapia de reposição de plasma tem grandes chances de desenvolver inibidores do fator XI (DIAMENT, 2004; FERREIRA et al., 2010).

Os indivíduos que possuem inibidores não sangram espontaneamente, mas possuem maior predisposição de sangramento durante e após cirurgia, mesmo quando há tratamento com plasma, já que os inibidores não permitem a ação do fator XI presente no plasma. Deve se suspeitar da presença de inibidores quando o tratamento utilizado não corrigir o TTPA. Em situações como essa e obrigatório a busca de inibidores através de testes como o da mistura (CONDE et al., 2007; CAVALCANTE, 2004). 
Pacientes com deficiência de fator XI podem ter hemorragia tanto durante a cirurgia quanto semanas mais tarde. Desse modo e' imprescindível o diagnostico antes da intervenção cirúrgica. Além disso, é necessário antes do tratamento, a pesquisa de inibidores, avaliar se o paciente possui outras doenças, como von Willebrand, o local e tipo de cirurgia, risco de sobrecarga e o risco trombótico. Existem diferentes tipos de tratamentos dependendo da intervenção a ser realizada no paciente (CONDE et al., 2007).

\section{Sinais e sintomas clínicos do paciente com hemofilia C}

Após sofrer um traumatismo, o paciente pode apresentar como os principais sinais e sintomas: hemorragias muco-cutôneas, equimose, sangramentos gengivais e epistaxe. Isso em maior ou menor grau, a depender do trauma e do grau da deficiência (FERREIRA et al., 2010; MINISTÉRIO DA SAÚDE, 2015).

Outros sintomas comuns presentes na deficiência do fator XI, são hematúria, menorragia e sangramento tardio em seu pós - operatório (DIAMENT, 2004).

\section{Hemofilia C: Quanto a gravidade}

Na deficiência severa, que e caracterizada pelo nível de atividade do fator XI no plasma inferior a $20 \%$ do normal, as manifestações hemorrágicas variam de acordo com o paciente com o tecido em que ocorreu a lesão (CAVALCANTE, 2004; DIEGO, 2007; EMILY et al., 2010).

Lesões em locais com alto potencial fibrinolítico, como mucosa oral, nasal e trato geniturinário, possuem maior probabilidade de hemorragia (49\% a $67 \%$ ) se comparado com locais com baixo potencial fibrinolítico, como músculos e articulações $(1,5 \%$ a $40 \%)$ (MODOLO et al, 2010; CONDE et al., 2007).

$\mathrm{Na}$ deficiência leve, onde a quantidade de fator XI no plasma varia de $30 \%$ a $65 \%$, somente e descrita a menorragia em mulheres, tanto homozigotos quanto heterozigotos (PEYVANDI et al., 2011).

\section{Tratamento médico para o paciente portador de hemofilia $\mathrm{C}$}

O plasma fresco congelado e' habitualmente usado e, possui eficácia durante as hemorragias e não aumenta a chance de desenvolver tromboses. Mas e' necessário grande quantidade para que haja efeito, já que a quantidade de fator XI no plasma e' bem pequeno. Além disso, existe risco de reações alérgicas, transmissão de agentes infecciosos e sobrecarga dos vasos sanguíneos devido ao aumento da volemia causado pela grande quantidade de plasma injetado. O tratamento sugerido pelo Ministério da Saúde é a reposição através do plasma, com dose inicial de $10 \mathrm{mg} / \mathrm{Kg}$ e dose de manutenção de $5 \mathrm{ml} / \mathrm{Kg}$ uma vez ao dia (MINISTÉRIO DA SAÚDE, 2015; CAVALCANTE, 2004). 
O concentrado de fator XI é indicado para deficiências mais severas. Precisa ser administrado com cuidado pois, pode induzir a formação de trombos. Eficaz em pequenos volumes, não causando sobrecarga no vaso sanguíneo (DIAMENT, 2004).

Os agentes antifibrinoliticos agem fazendo com que o coagulo permaneça formado, interrompendo a ação da plasmina. São eficaz no tratamento de menorragias e em pequenas cirurgias. Em caso de grandes cirurgias ou hemorragias intensas, não é eficaz (CONDE et al., 2007; DIAMENT, 2004; FERREIRA et al., 2010).

Os antifibrinoliticos utilizados são o acido tranexâmico e o acido épsilon aminocapróico, administrados por via oral ou intra - venosa (MINISTÉRIO DA SAÚDE, 2015; PEYVANDI et al., 2011).

A desmopressina é útil no tratamento quando ha deficiência simultânea de fator XI e o fator de von Willebrand. Ela age aumentando a concentração de fator FvW no plasma e, consequentemente aumentando a concentração do fator VIII, ja que o FvW impede a proteólise do fator VIII, administrada via nasal ou intra-venosa (PEYVANDI et al., 2011).

A cola de fibrina é extremamente eficaz no tratamento de feridas externas feitas por pequenas cirurgias, além de poder ser utilizada junto com o plasma fresco congelado. Porem não tem boa ação em grandes cirurgias e em hemorragias intensas (COPPOLA et al., 2013). O fator VII ativado recombinário é utilizado em indivíduos com deficiência do fator XI e presença de inibidores.

O fator VII ativado recombinante age junto com o fator tissular na iniciação da coagulação, ativando o fator X, que resultara na formação de fibrina (CONDE et al., 2007; MINISTÉRIO DA SAÚDE, 2015; COPPOLA et al., 2013).

\section{Como proceder no tratamento odontológico}

Pacientes com deficiência de fator XI podem ter hemorragia tanto durante a cirurgia quanto semanas mais tarde. Desse modo e imprescindível o diagnostico antes da intervenção cirúrgica (mesmo as cirurgias orais menores como exodontia e procedimentos periodontais evasivos). Além disso, e necessário, antes do inicio do tratamento, a pesquisa de inibidores, avaliar se o paciente possui outras doenças como de von Willebrand, o local e tipo de cirurgia, risco de sobrecarga e o risco trombótico (MINISTÉRIO DA SAÚDE, 2015; PEYVANDI et al., 2011; COPPOLA et al., 2013).

O plasma fresco congelado e habitualmente usado, possui eficácia durante as hemorragias e não aumenta a chance de desenvolver tromboses.

Mas é necessário grande quantidade para que haja efeito, já que a quantidade de fator XI no plasma e bem pequeno. Além disso, existe risco de reações alérgicas, transmissão de agentes infecciosos e sobrecarga dos vasos sanguíneos devido ao aumento da volemia causado pela grande quantidade de plasma injetado. O tratamento sugerido pelo Ministério da Saúde para a deficiência de fator XI, e a reposição através do plasma, com dose inicial de $10 \mathrm{mg} / \mathrm{kg}$ e dose 
COPYRIGHT @ 2019 INTERNATIONAL JOURNAL OF SCIENCE DENTISTRY | AVAILABLE ONLINE http://www.periodicos.uff.br/index

de manutenção de $5 \mathrm{ml} / \mathrm{Kg}$ uma vez ao dia (CONDE et al., 2007; DIAMENT, 2004; EMILY et al., 2010).

O concentrado de fator XI e indicado para deficiência mais severa. Precisa ser administrado com cuidado [pois, pode induzir a formação de trombos. E eficaz em pequenos volumes, não causando sobrecarga no vaso sanguíneo. Após preparação do paciente, podemos iniciar os procedimentos odontológicos (CONDE et al., 2007).

\section{Possíveis complicações do tratamento odontológico}

A principal complicação são as hemorrágicas. A primeira medida a ser tomada se houver sangramento e a limpeza e identificação da área sangrante e a remoção do coagulo formado. Depois de identificada a área sangrante, medidas locais de controle do sangramento devem ser tomadas, tais como:

- Sangramento pós- extração dentária

- Remoção da sutura e curetagem alveolar, posterior e realização de nova sutura;

- Aplicação do Soro físiológico 0,9\% (SF 0,9\%);

- Aplicação de outros meios hemostáticos locais, tais como antifibrinoliticos, etc.

- Sangramentos gengivais

- Pós - exfoliação de dente decíduo:

- Curetagem do tecido de granulação remanescente e sutura se possível;

- Aplicação de agentes hemostáticos locais, tais como SF 0,9\%, pasta de antifibrinoliticos e agentes hemostáticos odontológicos;

- Proteger a região com cimento cirúrgico ou splints;

- Sangramentos gengivais por outros motivos:

- Utilizar os agentes hemostáticos locais já descritos anteriormente; - Gelo no local.

- Traumas Mucosos

- Se possível, suturar o local e utilizar os agentes hemostáticos locais já descritos.

Em todos esses casos, o hematologista responsável deve ser consultado, visto que, em muitas das vezes, só o cuidado local não e suficiente para controle do sangramento e a terapia de reposição dos fatores de coagulação deve ser indicada. Nesses casos, a reposição dos fatores de coagulação deve seguir o protocolo proposto pelo Manual de Tratamento de Pacientes Portadores de Coagulopatias Hereditarias (DIAMENT, 2004; FERREIRA et al., 2010; PEYVANDI et al., 2011; PEYVANDI et al., 2011).

\section{DISCUSSÃO}


COPYRIGHT C 2019 INTERNATIONAL JOURNAL OF SCIENCE DENTISTRY | AVAILABLE ONLINE http://www.periodicos.uff.br/index

Embora a hemofilia seja conhecida desde a Antiguidade, seu tratamento só esta disponível ha poucas décadas (MODOLO et al., 2010; CONDE et al., 2007; DIEGO, 2007).

Muitas tentativas terapêuticas foram feitas, desde extirpação do baco, terapia de raiosx, compressas com veneno de cobra nas feridas ate transfusão de sangue mas, só nos últimos cinquentas anos e que apareceu trabalhos científicos que apresentavam formas de intenção no tratamento da Hemofilia (MODOLO et al., 2010; PEYVANDI et al., 2011; PEYVANDI et al., 2011). Hoje, muitos preconizam o tratamento de hemofilia na deficiência do Fator XI, com a infusão de Plasma Fresco Congelado, em doses de 15 a 20 ml / Kg para manter níveis mínimos na faixa dos $10 \%$ a $20 \%$. Como o FXI tem meia vida de 40 horas, a terapia de reposição pode ser administrada em dias alternados.

Pacientes com nível extremamente baixo de fator XI no plasma (cerca de 1UI / dL) expostos a terapia de reposição de plasma, tem grandes chances de desenvolverem inibidores do fator XI , que não permitem a ação do fator XI presente no plasma e, necessitam de grande quantidade para que haja efeito, já que a quantidade de fator XI no plasma e bem pequeno (MODOLO et al., 2010; CAVALCANTE, 2004; DIAMENT, 2004). Além disso, existe risco de reações alérgicas, transmissão de agentes infecciosos e sobrecarga dos vasos sanguíneos, devido ao aumento de volemia, causado pela grande quantidade de plasma injetado (CONDE et al., 2007; EMILY et al., 2010).

O concentrado de fator XI e indicado para deficiência mais severas, com o cuidado de não induzir a formação de trombos (CONDE et al., 2007; CAVALCANTE, 2004; DIEGO, 2007). Deve-se evitar o Plasma Fresco Congelado sempre que possível e principalmente em paciente hemofílico, pelo fato de estar disponível no Brasil, produtos mais seguros, com menos risco de transmissão viral. É proibido, pelo Ministério da Saúde, a utilização de plasma fresco congelado e criprecipitado em pacientes hemofílicos - RDC numero 23, de 24 de janeiro de 2002; DIEGO, 2007; EMILY et al., 2010; MINISTÉRIO DA SAÚDE, 2015; PEYVANDI et al., 2011).

A partir de 2015, o Ministério da Saúde passou a promover uma avançada na politica de atenção aos pacientes com coagulopatias hereditárias (CONDE et al., 2007; DIEGO, 2007; FERREIRA et al., 2010). Tratamento como a Imunotolerancia e profilaxia primaria, melhorando a qualidade de vida dos pacientes (FERREIRA et al., 2010; MINISTÉRIO DA SAÚDE, 2015; PEYVANDI et al., 2011).

Em 2012, o Ministério da Saúde, recomenda a profilaxia secundaria, que passaram a ser realizada de duas formas:

- Tratamento de Prevenção de Curta Duração :

Reposição do fator coagulante, administrando de maneira intermitente por tempo determinado (3 a 12 meses) para tratamento de sangramentos frequentes e complicações . Para qualquer tipo de hemofilia.

- Tratamento de Prevenção de Longa Duração : 
COPYRIGHT @ 2019 INTERNATIONAL JOURNAL OF SCIENCE DENTISTRY | AVAILABLE ONLINE http://www.periodicos.uff.br/index

Reposição administrada de maneira periódica e ininterrupta a longo prazo (45 semanas por ano), iniciado apos duas ou mais hemartroses ou apos a dois anos de idade. Só para hemofilia A ou B grave.

O tratamento adotado pelo Ministério de Saúde , em 2014, foi reconhecido pela Federação Mundial de Hemofila (MODOLO et al., 2010; CAVALCANTE, 2004). Fica estabelecido que, no caso de hemorragia intensa, não tendo o protocolo de tratamento, e feito em todos os casos, a aplicação do concentrado de fator de coagulação, para que permita que ela seja resolvida rapidamente (CONDE et al., 2007; DIEGO, 2007).

Em estudos uma edição do DNA, para consertar os trechos de genes que geram as falhas da hemofilia. (EMILY et al., 2010; FERREIRA et al., 2010; PEYVANDI et al., 2011). A terapia genica e aguardada para as próximas duas décadas (MODOLO et al., 2010; DIAMENT, 2004; EMILY et al., 2010; FERREIRA et al., 2010; PEYVANDI et al., 2011).

\section{CONCLUSÃO}

Uma anamnese, quando bem conduzida, é responsável por cerca de 85\% do diagnóstico no caso da deficiência de fator XI. Isso por que a mesma além de rara é de difícil identificação. $\mathrm{O}$ relato de hemorragias alguns dias após o procedimento cirúrgico, associado ao histórico familiar e descendência judaica Ashkenazi, se tornam de suma importância para um adequado plano de tratamento e a execução segura de procedimentos odontológicos.

\section{REFERÊNCIAS}

1. Modolo, NSP.; Azevedo, VLF.; Santos, PSS.; Rosa, ML.; Corvino, DR.; Alves, LJSC. Estratégias anestesiológicas para cesariana em paciente portadora de deficiência de fator XI: Relato de caso. Revista Brasileira de Anestesiologia. v. 60 n.2, Campinas, março / abril, 2010.

2. Conde, P.; Alegria, A.; Andradre, M. Deficiencia de Factor XI. Acta Medica, Portugal, Lisboa, 375- 380 v. 20 n. 4, 2007.

3. Cavalcante, SL. Sindrome de Rosenthal ou Hemofilia C: Relato de Caso. Revista Brasileira de Anestesiologia. Fortaleza v. 54, n. 33, 2004.

4. Diego, SS. O Fator XI Deficiency. Departamento of Biology and Genitics for Medical Sciencer: Seminars Thromboses and Hemostases. Milan, v 35, n. 8, 416 - 423, 2007.

5. Diament, J. Deficiência do Fator XI. Einstein. São Paulo. v.2, n.2 ,115 - 116, 2004.

6. Emily, T.; Macwan, PA.; Gailani, D. Structure and Function of Factor XI. Blood. Washington DC, V. 115 , N.13, 2369 - 2372, 2010. 
7. Ferreira, NC.; Souza, MO.; Dusse, LMS.; Carvalho, MG. O Novo Modelo da Cascata de Coagulação, baseado nas superfícies celulares e suas implicações. Revista Brasileira de Hematologia e Hemoterapia. São Paulo, 2010.

8. Ministério da Saúde. Manual de Hemofilia.. Brasil, 2015. http://bvsms.saude.gov.br/bvs/publicacoes/manual_hemofilia_2ed.pdf, visto em 06 de agosto de 2017, ‘as 22:05 hs.

9. Peyvandi, F.; Bidlingmaier, C.; Garagiola, I. Management of pregnancy and delivery in women with inherited bleeding disorders. Seminars in Fetal \& Neonatal Medicine, Amsterdam, v. 16, n. 6, p. 311-317, 2011.

10. Coppola, A. et al. Emerging issues on comprehensive hemophilia care: preventing, identifying, and monitoring age related comorbidities. Seminars in Thrombosis and Hemostasis, New York, v. 39, n. 7, p. 794-802, 2013. 